\title{
Dynamic nuclear polarization with an inhomogeneously broadened ESR line. II. Experiment*
}

\author{
David S. Wollan ${ }^{\dagger}$ \\ Physics Department, Virginia Polytechnic Institute and State University, Blacksburg, Virginia 24061
}

(Received 30 May 1974; revised manuscript received 30 October 1975)

\begin{abstract}
Results of proton dynamic nuclear polarization (DNP), nuclear spin-lattice relaxation, and ESR experiments in six YES: ${ }^{168} \mathrm{Er}\left({ }^{168} \mathrm{Er}\right.$-doped yttrium ethyl sulphate) single crystals at $4.15 \mathrm{~K}$ and $9.1 \mathrm{GHz}$ are reported here. This material approximates an inhomogeneously broadened (IHB) ESR line for certain crystal orientations relative to the applied magnetic field $\vec{H}_{0}$. DNP peak enhancements $E_{\mathrm{ss}}^{\text {peak }} \approx 120$ are obtained for crystals $1-3$ having ${ }^{168} \mathrm{Er}$ dilutions of $0.2 \%-0.01 \%$ (mole\%). The steady-state enhancement $E_{\mathrm{ss}}$ vs $H_{0}$ curves are proportional to the ESR absorption derivative at low powers $(\approx 0.3 \mathrm{~mW})$, but change shape in the wings of the ESR line at high microwave powers $(\approx 300 \mathrm{~mW})$. Essentially idential $E_{s s}$ data are obtained for all three crystals. Extensive DNP pump time $\tau_{\text {DNP }}$ and ESR data for crystals $2-4$, as well as limited data for the other crystals, are also given. There is $n o E_{\mathrm{ss}}$ or $\tau_{\mathrm{DNP}}$ evidence in these experiments (i) for the traditional strict-shellof-influence model of IHB SE (solid effect) DNP, (ii) for non-negligible CE (cross effect) DNP processes, or (iii) for decreases in $E_{\mathrm{ss}}$ due to moderate spectral diffusion in an IHB ESR line, as proposed by Buishvili et al. The DNP and ESR data of crystals 1-3 have been fit by the least-squares technique to a muffin-tin model of IHB SE DNP which incorporates rapid nuclear spin diffusion. Moderate agreement with theory is obtained, especially if the data are interpreted with a three-spectral-region model for wide IHB ESR lines. These data have also been analyzed in terms of EDDR (electron dipole-dipole reservoir) DNP theory for an IHB ESR line with fast spectral diffusion throughout the line. There is qualitative and some quantitative agreement between this theory and the data. It is thus difficult to discriminate EDDR DNP theory from IHB SE DNP with the three-spectral-region model in these experiments. The only clearcut evidence for the latter is an orderof-magnitude theoretical calculation of the electron magnetic dipole-dipole contribution to the ESR intrinsic spin-packet width, which gives non-negligible spectral diffusion in the centers of the crystal 1 and 2 ESR lines, and negligible spectral diffusion in the wings of crystals 3 and (perhaps) 2. Conclusive evidence would require further experiments at ${ }^{168} \mathrm{Er}$ dilutions lower than $0.01 \%$ and higher than $0.2 \%$. Experiments are proposed for YES: ${ }^{168} \mathrm{Er}$ to test further the three-spectral-region model, and to find high DNP by IHB SE with possible applications to polarized nuclear targets in other, more hydrogenous materials.
\end{abstract}

\section{INTRODUCTION}

Experiments on the dynamic nuclear polarization (DNP) of protons in erbium-doped yttrium ethyl sulphate (YES : Er) at $4.15 \mathrm{~K}$ and $9.1 \mathrm{GHz}$ are reported here, and interpreted with the theories developed in the preceding paper ${ }^{1}$ (denoted I, hereafter) whose notation is used throughout this paper. Preliminary experimental results on YES : Er have been given els ewhere. ${ }^{2-4}$ This material is useful for inhomogeneously broadened (IHB) DNP studies with unresolved satellites because of its large IHB linewidth ${ }^{5} \gtrsim 20$ Oe at $5^{\circ} \lesssim \theta$ $\lesssim 15^{\circ}$, where $\theta$ is the angle between $\overrightarrow{\mathrm{H}}_{0}$ and the $c$ axis of the axially symmetric YES : Er. In addition, Larson and Jeffries ${ }^{6}$ have measured the $T$ and $\theta$ dependence of $T_{e}$ in YES : Er at liquid-helium temperatures for the main ESR line and the ${ }^{167} \mathrm{Er}$ hyperfine lines. In particular, they found no evidence for a phonon bottleneck, which is a complicating factor in DNP analysis (see, e.g., Refs. 4, 5, and 7 in I).

In Sec. II, we summarize the experimental procedure. The experimental results are given and analyzed in terms of IHB solid-effect (SE) theory in Sec. III; this DNP analysis is based solely on Eqs. (14) and (15) of I. In Sec. IV, we discuss the difficulty of discriminating this
IHB SE DNP theory from electron dipole-dipole reservoir (EDDR) DNP theory in an IHB ESR line with fast spectral diffusion, even when considerable $E_{\text {ss }}, \tau_{\text {DNP }}$, and ESR data are available. The $\tau_{\mathrm{DNP}}$ and $T_{n}^{m}$ data are suggestive of the threespectral-region model proposed in Sec. V of $\mathrm{I}$. The summary is given in Sec. V.

\section{EXPERIMENTAL PROCEDURE}

Saturated YES : $x \%{ }^{168} \mathrm{Er}$ water solutions were prepared by the Erath ${ }^{7}$ method using $99.9999 \%$ rare-earth-purity yttrium oxide and electronicgrade barium ethyl sulphate. It is necessary to exclude ${ }^{167} \mathrm{Er}$ from the samples, ${ }^{5}$ so erbium oxide enriched to $99.987 \%$ in ${ }^{168} \mathrm{Er}$ was obtained from the Oak Ridge National Laboratory. The chemical formula for YES is $\mathrm{Y}\left(\mathrm{C}_{2} \mathrm{H}_{5} \mathrm{SO}_{4}\right)_{3} \cdot 9 \mathrm{H}_{2} \mathrm{O}$. The $0.2 \%$ dilution for crystal 1 was obtained from the ratio of the $\mathrm{Y}_{2} \mathrm{O}_{3}$ and ${ }^{168} \mathrm{Er}_{2} \mathrm{O}_{3}$ masses mixed together at the beginning of the chemical synthesis of YES : ${ }^{168} \mathrm{Er}$. Part of the YES : $0.2 \%{ }^{168} \mathrm{Er}$-saturated water solution obtained from this synthesis was diluted twice with a pure YES-saturated solution, to obtain solutions with $x=0.05 \%$ and $0.01 \%$. The volume ratios of the solutions being mixed determined the latter two dilution percentages. The reason for this procedure was the small amount 
TABLE I. Summary of experimental parameters for six YES : ${ }^{168} \mathrm{Er}$ crystals. ESR and DNP parameters are for $\theta=6.1^{\circ}$ and $T=4.15 \mathrm{~K}$.

\begin{tabular}{|c|c|c|c|c|c|c|}
\hline Crystal & 1 & 2 & 3 & 4 & $5^{a}$ & 6 \\
\hline Mass (mg) & 188 & 129 & 233 & 161 & $\cdots$ & $\cdots$ \\
\hline$\%^{168} \operatorname{Er}(\text { Sol. })^{b}$ & 0.2 & 0.05 & 0.01 & 0.01 & 0.05 & $\gtrsim 1.0$ \\
\hline$\%{ }^{168} \operatorname{Er}(\text { crystal })^{c}$ & 0.033 & 0.011 & 0.0014 & $\cdots$ & ... & $\cdots$ \\
\hline$\nu_{e}(\mathrm{GHz})$ & 9.125 & 9.160 & 9.085 & 9.142 & 9.144 & 9.085 \\
\hline$H_{00}(\mathrm{Oe})$ & 3702 & 3701 & 3702.5 & 3700 & 3699 & $3720^{d}$ \\
\hline$\Delta H_{p p}(\mathrm{Oe})$ & 25.0 & 19.25 & 24.0 & 21.0 & $\cdots$ & 45 \\
\hline$T_{n}^{m}(\mathrm{sec})$ & 1.45 & 8.36 & 193 & 187 & $\cdots$ & $\ldots$ \\
\hline$f_{e}^{m}$ & $0.91^{\mathrm{e}}$ & $0.63^{\circ}$ & $0.14^{e}$ & ... & ... & ... \\
\hline
\end{tabular}

of ${ }^{168} \mathrm{Er}_{2} \mathrm{O}_{3}$ available (cost $\approx \$ 5$ per mg). All dilutions in this paper are in mole percent. Single crystals were grown from these saturated water solutions at $0{ }^{\circ} \mathrm{C}$, and the nomenclature for the crystals labeled $1-6$ is given in Table I. Crystal 6 was grown from an earlier batch whose dilution was not well measured, but was probably $x \gtrsim 1 \%$. The row labeled $\%{ }^{168} \operatorname{Er}($ Sol. ) in Table I gives the ${ }^{168} \mathrm{Er}$ concentrations measured as above. The ${ }^{168} \mathrm{Er}$ dilutions for crystals $1-3$ were also measured by an ESR technique; these concentrations are labeled $\%^{168} \operatorname{Er}$ (crystal).

Conventional ESR, NMR, and cryogenic techniques were used. ${ }^{4,5,8,9}$ An $X$-band crystal-video reflection spectrometer was used either to measure ESR spectra or to excite the DNP with variable microwave power up to a maximum $\approx 0.3 \mathrm{~W}$ in the cavity. The proton DNP, excited at fixed microwave frequency $\nu_{e}$ and variable $H_{0}$, was measured from the ratio of the proton NMR signal peak-to-peak height when polarized to that at thermal equilibrium. $Q$-meter ${ }^{5,8,9}$ and lock-in detection were used with magnetic field modulation at $f_{m}=97 \mathrm{~Hz}$. Because of the poor thermalequilibrium proton NMR signal, our DNP measurements have an absolute accuracy of $\pm 20 \%$. The relative accuracy of the DNP for $E_{s s}>10$ is $\pm 5 \%$. The relative accuracies of measurements of the proton spin-lattice relaxation time $T_{n}^{m}$ and the DNP pump time $\tau_{\text {DN }}$ vary from $\pm 3 \%$ to $\pm 10 \%$.

\section{EXPERIMENTAL RESULTS AND ANALYSIS}

\section{A. ESR results}

Previous work ${ }^{5}$ on YES : Er showed an anomalous DNP effect associated with the overlap of the main erbium ESR line with one of the ${ }^{167} \mathrm{Er}$ hyperfine lines. This anomaly was observed below $T \approx 2 \mathrm{~K}$, but not at $T=4.2 \mathrm{~K}$. Since we wished to avoid the anomaly in these experiments, we took the twin precautions of using YES : ${ }^{168} \mathrm{Er}$ with erbium enriched to $99.987 \%{ }^{168} \mathrm{Fr}$ and of operating at $T=4.2$ K. Since the ratio $T_{e} / T_{2 e}^{B}$ is lower at $4.2 \mathrm{~K}$ than, e.g., at $2.0 \mathrm{~K}$, this also tends to decrease the probability of EDDR DNP relative to IHB SE DNP.

The main ESR line in YES : Er, due to the eveneven Er isotopes, has axial symmetry and principal electron $g$ values ${ }^{5,6} g_{11}=1.503$ and $g_{\perp}=8.770$. The ESR linewidth $\Delta H_{p p}$, defined to be the peakto-peak width of the ESR absorption derivative, has a maximum of $\Delta H_{p p} \approx 20-30$ Oe at $\theta \approx 15^{\circ}$, falling off to $\Delta H_{p p} \approx 5-8$ Oe as $\theta$ goes to $0^{\circ}$ and to $90^{\circ} .5$ Measurements of $\Delta H_{p p}$ as a function of $\theta$ for crystal 3 at $T=4.15 \mathrm{~K}$ are shown in Fig. 1 . The data have been fit by the least-squares tech-

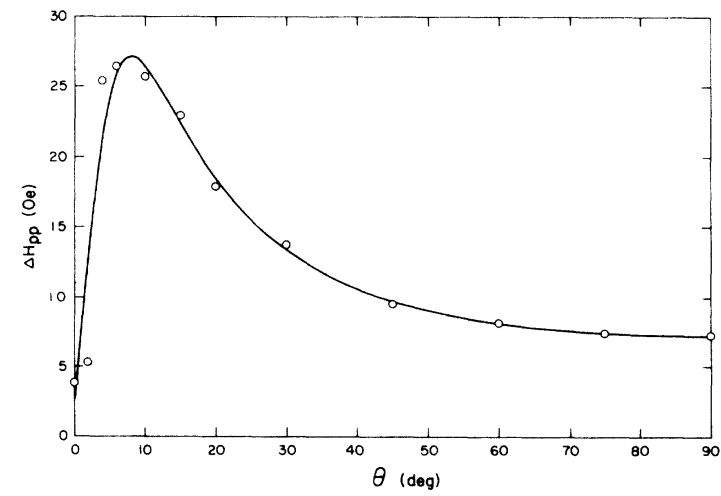

FIG. 1. Measured ESR derivative peak-to-peak linewidth $\Delta H_{p p}$ vs orientation angle $\theta$ for crystal 3 at $4.15 \mathrm{~K}$. Solid line is Eqs. (1) and (2), using $K=4$ and the leastsquares fit parameters given in the text. 


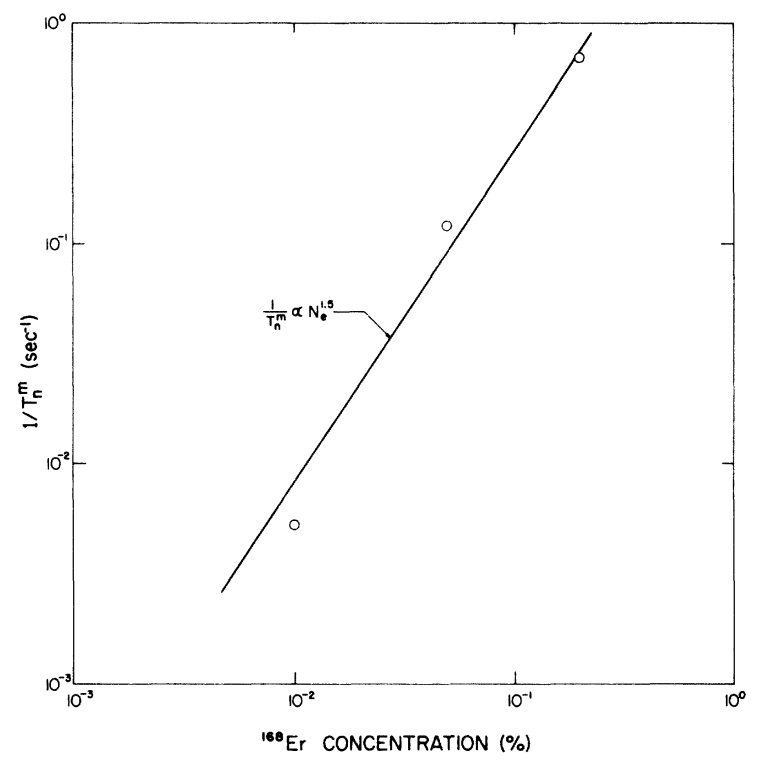

FIG. 2. Measured proton spin-lattice relaxation rate $\left(T_{n}^{m}\right)^{-1}$ vs ${ }^{168} \mathrm{Er}$ concentration for crystals 1,2 , and 3 at $\theta=6.1^{\circ}, H_{0}=3715 \mathrm{Oe}$, and $T=4.15 \mathrm{~K} .{ }^{168} \mathrm{Er}$ concentration is $\%^{168} \mathrm{Er}$ (Sol.) given in Table I.

nique to the IHB theoretical linewidth formula of Scott et al. ${ }^{10}$ :

$$
\Delta H_{\mathrm{pp}}=\left(K\left\langle\delta H^{2}\right\rangle\right)^{1 / 2},
$$

where

$$
\begin{aligned}
\left\langle\delta H^{2}\right\rangle= & \left\langle\delta H_{d}^{2}\right\rangle+\left[h \nu_{e} / g_{e}^{3}(\theta) \mu_{B}\right]^{2}\left[g_{\|}^{2} \cos ^{4} \theta\left\langle\delta g_{\|}^{2}\right\rangle\right. \\
& \left.+g_{\perp}^{2} \sin ^{4} \theta\left\langle\delta g_{1}^{2}\right\rangle+\left(g_{1}^{2}-g_{\|}^{2}\right)^{2} \cos ^{2} \theta \sin ^{2} \theta\left\langle\delta \theta^{2}\right\rangle\right] .
\end{aligned}
$$

For a Gaussian line shape $K=4$ holds. The angularindependent term $\left\langle\delta H_{d}^{2}\right\rangle$ is the square of the local magnetic field at the electron site due to neighboring nuclear magnetic moments. The angulardependent terms $\left\langle\delta g_{\Perp}^{2}\right\rangle,\left\langle\delta g_{1}^{2}\right\rangle$, and $\left\langle\delta \theta^{2}\right\rangle$, all assumed independent of one another, are variations of $g_{11}, g_{1}$, and $\theta$ at various sites in the crystal due to, e.g., crystal-axis wander, mosaic structure, and lattice strains. The remaining symbols in Eqs. (1) and (2) are defined above or in I, except for Planck's constant $h$. In Fig. 1 the solid line is Eqs. (1) and (2) using $K=4$ and the best-fit parameters $\left\langle\delta H_{d}^{2}\right\rangle=1.46,\left\langle\delta g_{11}^{2}\right\rangle=5.7 \times 10^{-8}$, $\left\langle\delta g_{1}^{2}\right\rangle=1.63 \times 10^{-3}$, and $\left\langle\delta \theta^{2}\right\rangle=1.65 \times 10^{-6}$. We note that $\left(K\left\langle\delta H_{d}^{2}\right\rangle\right)^{1 / 2} \approx 2.4$ Oe for $K=4$, so that the peak in $\Delta H_{\mathrm{pp}}$ near $\theta \approx 10^{\circ}$ is due to the crystal-axis wander, mosaic-structure, etc., terms and not to $\left\langle\delta H_{d}^{2}\right\rangle$ or to homogeneous broadening (HB) interactions. Thus it is probable that the electron spin-packet jump time $\tau$ satisfies $\tau \gg T_{n}^{m}$ in YES : ${ }^{168} \mathrm{Er}$ at $\theta \approx 5^{\circ}-10^{\circ}$. Essentially identical data and fits to Eqs. (1) and (2) were made for crystals 1 and 2 and are not reproduced here.
The ESR absorption derivative peak-to-peak amplitudes of crystals $1-3$ at $\theta=45^{\circ}$ were used in comparison with those of a phosphorous-doped silicon ESR standard sample ${ }^{11}$ to measure the ${ }^{168} \mathrm{Er}$ dilutions in those three crystals, taking into account the anisotropy of the electron $g$ factor. ${ }^{12}$ Because of possible ESR saturation effects and uncertain numerical factors due to line-shape differences, the ${ }^{168} \mathrm{Er}$ dilutions measured by the ESR calibration $\left[\%^{168} \mathrm{Er}\right.$ (crystal) in Table I] may be off by a factor $\approx 2-3$. The ratio $\%{ }^{168} \operatorname{Er}($ Sol. $) /$ $\%^{168} \operatorname{Er}$ (crystal) is found to be 6.0,4.7, and 7.1 for crystals 1,2 , and 3 , respectively. We have arbitrarily deduced $N_{e}$ for the ${ }^{168} \mathrm{Er}$ ions in crystals 1-3 from the $\%{ }^{168} \operatorname{Er}(\mathrm{Sol}$. ) row in Table I, with the understanding that $N_{e}$ may be high by a factor $\approx 2-6$ as a result.

\section{B. Nuclear spin-lattice relaxation results}

No systematic study of $T_{n}^{m}$ in YES : ${ }^{168} \mathrm{Er}$ was made. Instead, $T_{n}^{m}$ was measured only under the conditions of the DNP experiments (Table I). The rate $1 / T_{n}^{m}$ is plotted vs ${ }^{168} \mathrm{Er}$ concentration for crystals $1-3$ in Fig. 2. We see that $1 / T_{n}^{m}$ is approximately proportional to $N_{e}^{1.5}$, where the exact value 1.5 of the exponent may not be significant. This result does tell us that $T_{n}^{m}$ does not equal $T_{n}^{Z}$ for ${ }^{13}\left(1 / T_{n}^{Z}\right)=\left(\epsilon^{2} / \omega_{n}^{2}\right)\left(1 / T_{e}\right) \propto N_{e}$. Hence some electron spin-spin effects are acting on $T_{n}^{m}$, either by cross effect (CE) or by EDDR.

\section{DNP results}

The principal DNP, $T_{n}^{m}$, and ESR parameters of six YES : ${ }^{168} \mathrm{Er}$ crystals at $T=4.15 \mathrm{~K}$ are summarized in Table I. The ESR line center $H_{00}$ was set at $H_{00} \approx 3700$ Oe by adjusting the crystal angle to $\theta=6.1^{\circ}$, a convenient but arbitrary choice near the peak of the $\Delta H_{p p}$ vs $\theta$ curve of, e.g. , Fig. 1 . The idea was to maximize the IHB contribution to the ESR linewidth. The measured values of $\Delta H_{p p}$ and $T_{n}^{m}$ are given in Table $\mathrm{I}$, as is the measured DNP leakage factor $f_{e}^{m}=N_{n} T_{e} / N_{e} T_{n}^{m}$, where $T_{e}$ $\left(=8 \times 10^{-5} \mathrm{sec}\right)$ is taken from Ref. 6 , and $N_{e} / N_{n}$ is obtained from $\%^{168} \mathrm{Er}$ (Sol.). No $T_{e}$ values were measured for these crystals. Under the conditions of Table I, the ideal enhancement $E_{i}$ is 577 , and the satellite separation $\left(H_{+}-H_{-}\right)$is $12.8 \mathrm{Oe}$, corresponding to $\omega_{n}=6.4 \mathrm{Oe}$.

The measured peak enhancements $E_{\mathbf{s s}}^{\text {peak }}$ for crystals 1-6 at full microwave power are given in Table $I$ as a function of the magnetic field modulation peak-to-peak depth $H_{m}$. None of the $E_{\mathbf{s s}}^{\text {peak }}$ was limited by insufficient microwave power. Within the $\pm 20 \%$ absolute accuracy, $E_{s s}^{\text {peak }}$ is the same for crystals 1-4 for all $H_{m}$ values. Crystal 5 was badly cracked, and no further experiments were done on it. Crystal 6 was the first crystal run, at a time when the instrumentation was not per- 
TABLE II. Peak enhancements $E_{s s}^{\text {peak }}$ measured in six crystals for different values of $H_{m}$ under the conditions of Table I at full microwave power.

\begin{tabular}{ccccccc}
\hline \hline$H_{m}(\mathrm{Oe})$ & Crystal 1 & Crystal 2 & Crystal 3 & Crystal 4 & ${\text { Crystal } 5^{\mathrm{a}}}^{2}$ & Crystal 6 \\
\hline 4.0 & 117 & 113 & 135 & 129 & $72^{\mathrm{a}}$ & $\ldots$ \\
1.5 & 107 & 113 & 125 & $\ldots$ & $\ldots$ & $\approx 19^{\mathrm{b}}$ \\
0.5 & 108 & $\ldots$ & $\ldots$ & $\ldots$ & $\ldots$ & $\ldots$ \\
\hline \hline
\end{tabular}

${ }^{\mathrm{a}}$ Cracked crystal; see text.

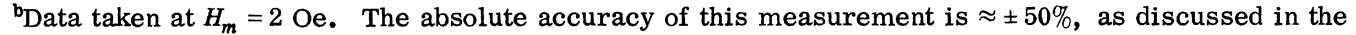
text.

fected, and we observed a low $E_{\mathbf{s s}}^{\text {peak }} \approx 19$ with a large absolute error $\approx \pm 50 \%$. The shape of the $E_{\text {ss }}$ vs $H_{0}$ curve changed with varying microwave power. This was undoubtedly EDDR DNP. Since our main interest was in IHB DNP, new batches of YES : ${ }^{168} \mathrm{Er}$ crystals were grown at lower ${ }^{168} \mathrm{Er}$ dilutions.

In all cases, the proton NMR signal growths and decays as a function of time could be leastsquares fit to a single exponential, and the measured $\tau_{\mathrm{DNP}}$ and $T_{n}^{m}$ values were determined as the best-fit parameters. No extensive $\tau_{D N P}$ or ESR data were taken in crystal 1 ; a single value of $\tau_{\mathrm{DNP}}^{\infty}=0.65 \mathrm{sec}$ was measured in crystal 1 at $P=0 \mathrm{~dB}$ at the positive $E_{\mathrm{ss}}$ enhancement peak $\left(H_{0}\right.$ $=3713 \mathrm{Oe})$ with $H_{m}=4 \mathrm{Oe}$. $\quad\left(\tau_{\mathrm{DNP}}^{\infty}\right.$ will be defined below.) In some cases (see Figs. 10 and 11), $\tau_{\text {DN P }}$ and ESR data were not taken for crystal 3 . However, such data were taken for crystal 4 and then used in the crystal 3 least-squares fit, since the $N_{e}, \Delta H_{p p}$, and $T_{n}^{m}$ values of crystals 3 and 4 are essentially the same (see Table I). By adjusting several microwave attenuators in the ESR system, it was possible to vary the microwave cavity power $P$, while letting the ESR crystal detector power remain constant. Thus we measured the relative ESR signal as a function of $P$ for crystals 2 and 4 . Within experimental error, the measured $\Delta H_{p p}$ of the ESR lines was a constant for each crystal at all powers measured, indicative of an IHB line, but possibly not telling whether fast spectral diffusion was present. The amplitudes $Y_{\mathrm{ESR}}$ from these measurements (Fig. 11) are discussed below.

Extensive $E_{\mathrm{ss}}, \tau_{\mathrm{DNP}}$, and ESR measurements were made on crystals 1-3, and least-squares fit to the IHB SE DNP theory given in Sec. IIC of I. Because the absolute value of $W^{ \pm}$was not measured, Eqs. (14) and (15) in I were transformed to the forms

$$
\begin{aligned}
& E_{\mathrm{ss}}=\frac{P_{1 / 2}+P\left(f_{e}^{m} / \xi\right)+S_{F}\left(E_{i}\right)(P)\left[G\left(H_{-}\right)-G\left(H_{+}\right)\right]}{P_{1 / 2}+P\left[G\left(H_{+}\right)+G\left(H_{-}\right)+f_{e}^{m} / \xi\right]}, \\
& \tau_{\mathrm{DNP}}=T_{n}^{m} \frac{S_{F}^{*}\left(P_{1 / 2}\right)+P\left(f_{e}^{m} / \xi\right)}{S_{F}^{*}\left(P_{1 / 2}\right)+P\left(f_{e}^{m} / \xi\right)+S_{F}(P)\left[G\left(H_{+}\right)+G\left(H_{-}\right)\right]} .
\end{aligned}
$$

Using a rectangular spin-packet model for a completely IHB ESR line, it is easy to show

$$
Y_{\mathrm{ESR}}=Y_{\mathrm{ESR}}^{\max } \frac{P_{1 / 2}}{P_{1 / 2}+P / S_{F 6}(\xi)},
$$

where $Y_{\text {ESR }}$ is the measured ESR absorption derivative peak-to-peak amplitude (in arbitrary units), and $Y_{\mathrm{ESR}}^{\max }$ is the completely unsaturated $(P=0)$ value. In these equations, $P$ is the microwave power in units where $P=1$ is the maximum power ( $\approx 300 \mathrm{~mW}$ in the cavity). Often the power $P$ is given in $\mathrm{dB}$, e.g., $P=1=0 \mathrm{~dB}$ or $P=10^{-3}=-30$ dB. Sometimes we shall refer to, e.g., $P=10^{-3}$ as 30-dB microwave attenuation. Since $S=W^{ \pm} T_{n}$ $\propto 1 / \xi$ for a spin packet in an IHB ESR model, we have transformed $S$ in Eqs. (14) and (15) of I to $P / \xi$ in Eqs. $(3)-(5)$ here. $P_{1 / 2}$ is the half-power parameter. We use the measured $T_{n}^{m}$ and $f_{e}^{m}$ from Table I in Eqs。(3) and (4).

Six sets of data (except as noted) for each of crystals 1-3 were simultaneously least-squares fit to Eqs. (3)-(5) as follows: (i) Measurements of $E_{\text {ss }}$ vs $H_{0}$ at $P=0 \mathrm{~dB}$ were fit to Eq. (3) with $S_{F}=1.0$. (ii) $E_{\text {ss }}$ vs $H_{0}$ data at $P=-30 \mathrm{~dB}$ were fit to Eq. (3) with $S_{F}$ redefined to be $S_{F 1}$. (iii) $E_{\text {ss }}$ vs $P$ data at the positive enhancement peak were fit to Eq. (3) with $S_{F}$ redefined to be $S_{F 2}$. (iv) Measurements of $\tau_{\mathrm{DNP}}$ vs $H_{0}$ at $P=0 \mathrm{~dB}$ were fit to Eq. (4) with $S_{F}^{*}=1.0$ and $S_{F}$ redefined to be $S_{F 3}$. (v) $\tau_{\mathrm{DNP}}$ vS $P$ data at the positive enhancement peak were fit to Eq. (4) with $S_{F}$ redefined to be $S_{F 4}$ and $S_{F}^{*}$ redefined to be $S_{F 5}$. (vi) Measurements of $Y_{\text {ESR }}$ vs $P$ were fit to Eq. (5) with the scale factor $S_{F 6}$ as is. The purpose of introducing the scale factors $S_{F 1}-S_{F 6}$, which we had initially hoped would be $\approx 1.0 \pm 0.20$, is to facilitate comparis on of the data points with curves plotted from Eqs. (3)-(5) using the best-fit parameters. Since the measured ESR $d G / d H$ is very close to a Gaussian, we have used a Gaussian $G(H)$ normalized to unity with a width determined by the experimental ESR $\Delta H_{p p}$. Equation (3) gives essentially identical results with $G(H)$ either Gaussian or numerically integrated from the experimental $d G / d H$, even in the wings of the ESR line. 


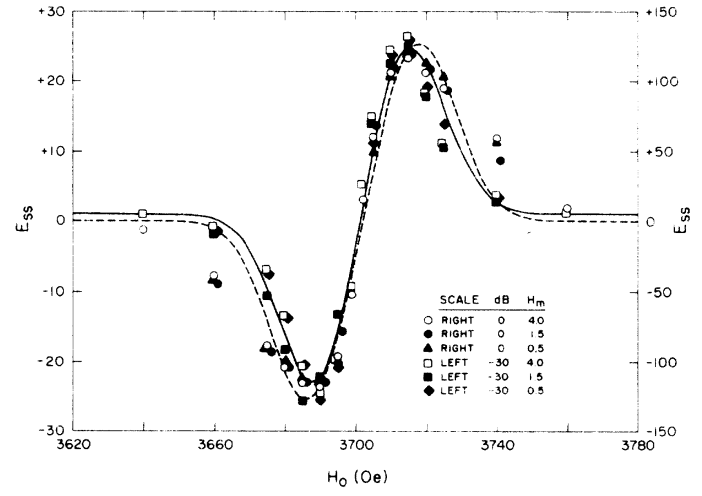

FIG. 3. Enhancement $E_{\mathbf{s s}}$ vs $H_{0}$ for crystal 1 at two microwave attenuator settings and three $H_{m}$ values. Solid (broken) line is Eq. (3) with $P=0.001$ (1.0) using the best-fit parameters in Table III. Solid data points were not used in the least-squares fit; relevant scale factors are given in Table IV.

DNP and ESR data points are plotted for crystals 1-4 in Figs. 3-11. The lines in those figures are Eqs. (3)-(5) using the best-fit parameters in Table III. The least-squares fit to Eqs. (3)-(5) used only the open data points in Figs. 3-11. For comparison purposes, other data have been plotted as solid points, but were not used in the fit. Some of the $E_{\text {ss }}$ data were scaled as in Table IV before being plotted as solid data points in Figs. 3-7. The scale factors in Table IV all lie between 0.95 and 1.14 , well within $\pm 20 \%$ of 1.0 , except for the 1.49 value.

We now examine the best-fit parameters in Table III. The first point to emphasize is that all six sets of data [except only sets (i)-(iii) for

TABLE III. Best-fit parameters determined from a least-squares fit of the data to Eqs. (3)-(5) in the text for crystals 1,2 , and 3 . See text for discussion of the scale factors $S_{F 1}$ to $S_{F 6}$.

\begin{tabular}{lcll}
\hline & Crystal 1 & Crystal 2 & ${\text { Crystal } 3^{\mathrm{a}}}$ \\
\hline$\xi(\mathrm{Oe})$ & 18.91 & 7.73 & 3.19 \\
$P_{1 / 2}$ & 0.00030 & 0.00048 & 0.00048 \\
$Y_{\mathrm{ESR}}^{\max }$ & $\ldots$ & 215.7 & 177.8 \\
$S_{F 1}$ & 0.88 & 0.96 & 1.36 \\
$S_{F 2}$ & 0.93 & 0.89 & 0.99 \\
$S_{F 3}$ & $\ldots$ & 8.36 & 9.18 \\
$S_{F 4}$ & $\ldots$ & 1.47 & 3.63 \\
$S_{F 5}$ & $\ldots$ & 4.47 & 8.83 \\
$S_{F 6}$ & $\ldots$ & 0.54 & 1.77 \\
\hline \hline
\end{tabular}

${ }^{a}$ Experimental data for crystal 4 are used when no equivalent crystal-3 data were taken, i.e., for $\tau_{\mathrm{DNP}}$ vs $P$ (Fig. 10) and for $Y_{\mathrm{ESR}}$ vs $P$ (Fig. 11).
TABLE IV. Scale factors for plotting $E_{\mathrm{ss}}$ data points in Figs. 3-7 for comparison purposes. Scale factors of all data points are 1.00 unless given in this table. None of the solid data points scaled using the values below was used in the least-squares fits of Eqs. (3)-(5) in the text.

\begin{tabular}{ccccc}
\hline \hline Fig. & Crystal & $H_{m}(\mathrm{Oe})$ & $\begin{array}{l}\text { Microwave } \\
\text { power }(\mathrm{dB})\end{array}$ & $\begin{array}{l}\text { Scale } \\
\text { factor }\end{array}$ \\
\hline 3 & 1 & 1.5 & 0 & 1.11 \\
3 & 1 & 0.5 & 0 & 1.10 \\
3 & 1 & 1.5 & -30 & 1.49 \\
3 & 1 & 0.5 & -30 & 0.99 \\
4 & 1 & 1.5 & $\ldots$ & 1.10 \\
4 & 1 & 0.5 & $\ldots$ & 1.14 \\
5 & 3 & 1.5 & 0 & 1.07 \\
6 & 3 & 1.5 & -30 & 0.95 \\
7 & 2 & 1.5 & $\ldots$ & 1.05 \\
\hline \hline
\end{tabular}

crystal 1] for each crystal were simultaneously least-squares fit to Eqs.(3)-(5). Thus, e.g., the parameters in the crystal- 2 column of Table III are based on all the crystal-2 $E_{s s}, \tau_{\mathrm{DNP}}$, and $Y_{\text {ESR }}$ open data points in Figs. 5-7 and 9-11. Second, of the three nonscale factor parameters in Table III, only $\xi$ is physically interesting. $P_{1 / 2}$ would be interesting if the microwave power in the cavity were accurately known, but no attempt was made to measure it. It is only significant that $P_{1 / 2}$ is the same within $50 \%$ for crystals 1-3. $Y_{\mathrm{ESR}}^{\max }$ is just the unsaturated ESR amplitude. Thus, excluding $S_{F 1}-S_{F 6}$, this least-squares fit is essentially a one-parameter fit as far as physically interesting quantities go. Third, we consider the scale factors $S_{F 1}-S_{F 6}$. The first two, $S_{F 1}$ and $S_{F 2}$, which relate to $E_{\mathrm{ss}}$ data, lie between 0.88 and 0.99 for all three crystals, ex-

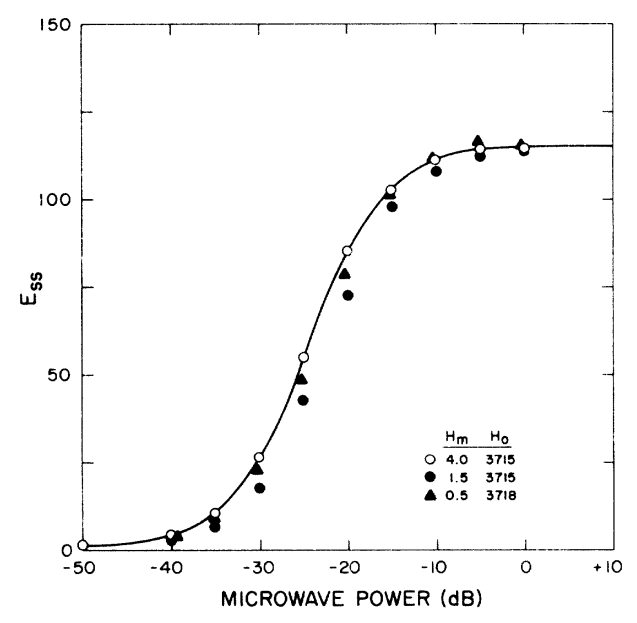

FIG. 4. Enhancement $E_{\text {ss }}$ vs microwave power for crystal 1 at $H_{0} \approx 3715$ Oe and three $H_{m}$ values. Solid line is Eq. (3) using the best-fit parameters in Table III. Solid data points were not used in the least-squares fit; relevant scale factors are given in Table IV. 


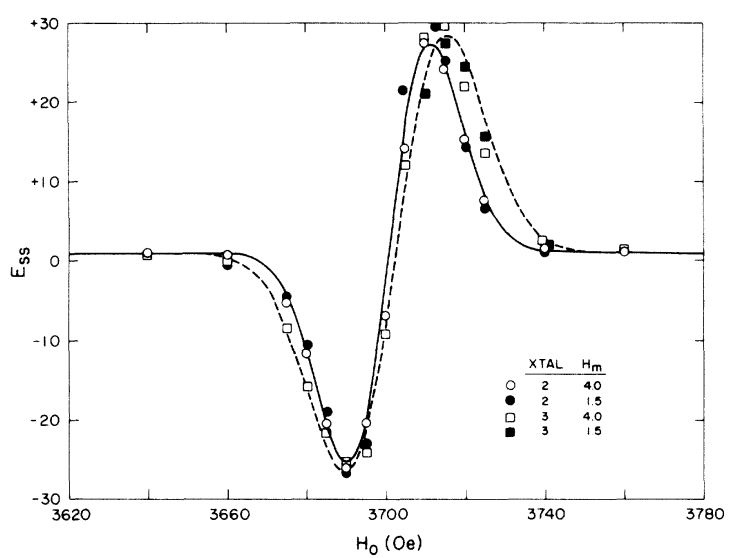

FIG. 5. Enhancement $E_{\mathbf{s s}}$ vs $H_{0}$ for crystals 2 and 3 at 30-dB microwave attenuation and two $H_{m}$ values. Solid (broken) line is Eq. (3) for crystal 2 (3) with $P=0.001$ using the best-fit parameters in Table III. Solid data points were not used in the least-squares fit; relevant scale factors are given in Table IV.

cept for the $S_{F 1}$ of 1.36 for crystal 3 , which is barely significant. The scale factors $S_{F 3}-S_{F 5}$ $\approx 1.5-9.2$, which relate to $\tau_{\mathrm{DN}}$, are a real puzzle. The least-squares program was run about 30 times, often varying more or fewer parameters than those in Table III. The resulting fits were either worse or essentially the same as those reported here. It was not possible to vary a combination of scale factors and parameters in such a way as to make $S_{F 3}-S_{F 5}$ all be $1.0 \pm 0.2$. The final best fit in Table III was not sensitive to the starting values of the parameters or to the order in which they were varied. The ESR scale factor $S_{F 6}$ is within a factor of 2 of unity, but is not

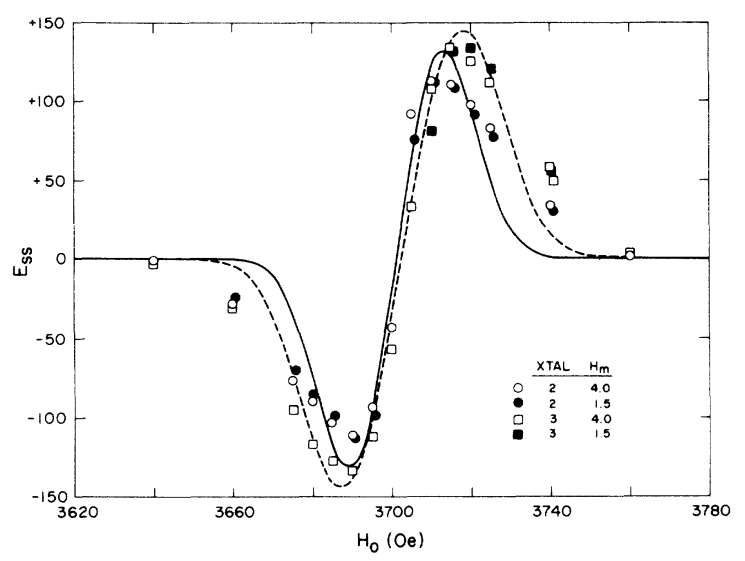

FIG. 6. Enhancement $E_{\mathbf{s s}}$ vs $H_{0}$ for crystals 2 and 3 at $0-\mathrm{dB}$ microwave attenuation and two $H_{m}$ values. Solid (broken) line is Eq. (3) for crystal 2 (3) with $P=1.0$ using the best-fit parameters in Table III. Solid data points were not used in the least-squares fit; relevant scale factors are given in Table IV.

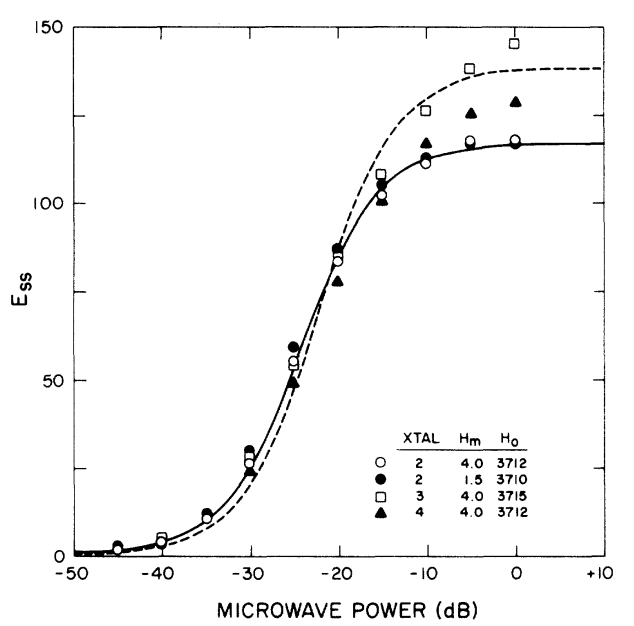

FIG. 7. Enhancement $E_{\mathbf{s s}}$ vs microwave power for crystals 2,3 , and 4 at $H_{0} \approx 3712$ Oe. Solid (broken) line is Eq. (3) for crystal 2 (3) using the best-fit parameters in Table III. Solid data points were not used in the leastsquares fit; relevant scale factors are given in Table IV.

consistently $<1.0$ as is expected. ${ }^{4}$

Let us now examine Figs. 3-11 in detail. Except as noted, all the shapes of Eqs. (3)-(5) in the figures fit the data points well. All the data points are independent of $H_{m}$ in these figures as in Table II. The $P=-30-\mathrm{dB} E_{\text {ss }}$ data in Figs. 3 and 5 fit Eq. (3) well, thus validating the Gaussian approximation for $G(H)$. However, the $P=0-\mathrm{dB}$ $E_{\text {ss }}$ data in Figs. 3 and 6 fall off less rapidly in the wings of the line than do the Eq. (3) lines. The change in the shape of $E_{\mathrm{ss}}$ vs $H_{0}$ for crystal 1 in Fig. 3 as $P$ goes from $-30 \mathrm{~dB}$ to $0 \mathrm{~dB}$, is far

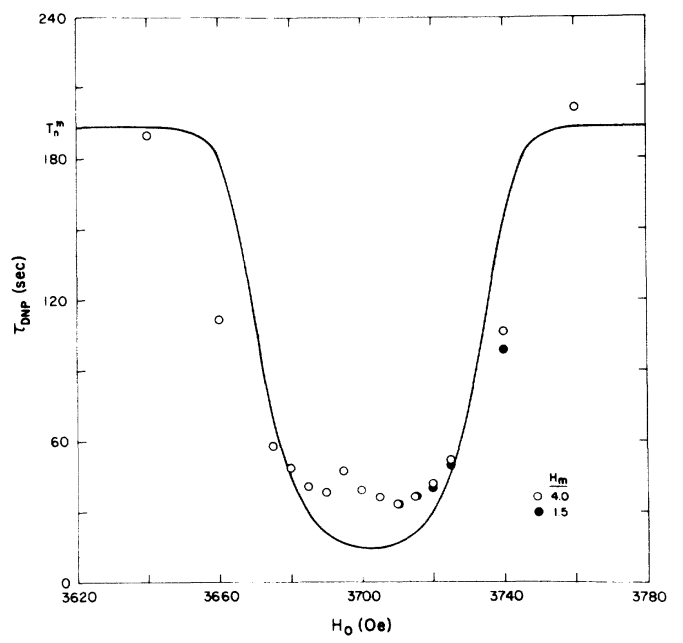

FIG. 8. DNP pump time $\tau_{\text {DNP }}$ vs $H_{0}$ for crystal 3 at $0-\mathrm{dB}$ microwave attenuation and two $H_{m}$ values. Solid line is Eq. (4) with $P=1.0$ using the best-fit parameters in Table III. Solid data points were not used in the leastsquares fit. 


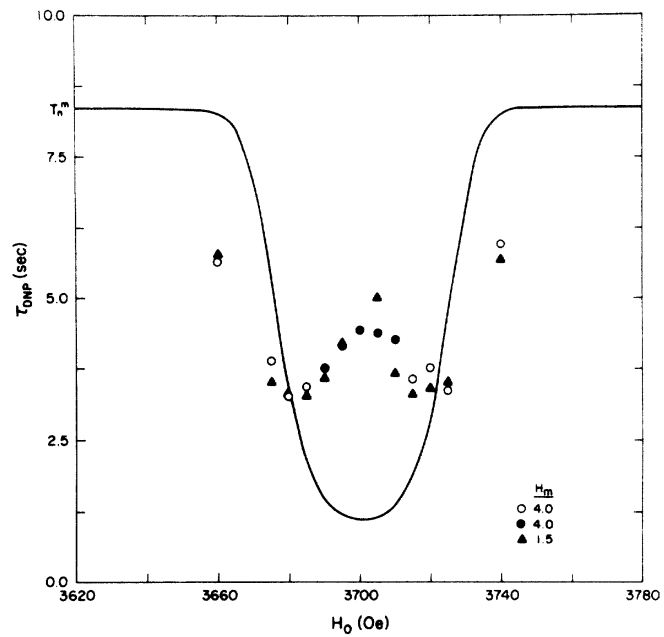

FIG. 9. DNP pump time $\tau_{\text {DNP }}$ vs $H_{0}$ for crystal 2 at $0-\mathrm{dB}$ microwave attenuation and two $H_{m}$ values. Solid line is Eq. (4) with $P=1.0$ using the best-fit parameters in Table III. Solid data points were not used in the leastsquares fit.

greater for the data points than for Eq. (3). A similar effect would be seen if Figs. 5 and 6 were overlaid. The crystal-2 and $-3 E_{\text {ss }}$ data at -30 dB in Fig. 5 are ess entially identical, aside from the small differences due to differing $\Delta H_{p p}$, and similarly for the 0-dB $E_{\text {ss }}$ data in Fig. 6. Adding the crystal-1 data from Fig. 3 to Figs. 5 and 6 would not change this conclusion. Hence, for both

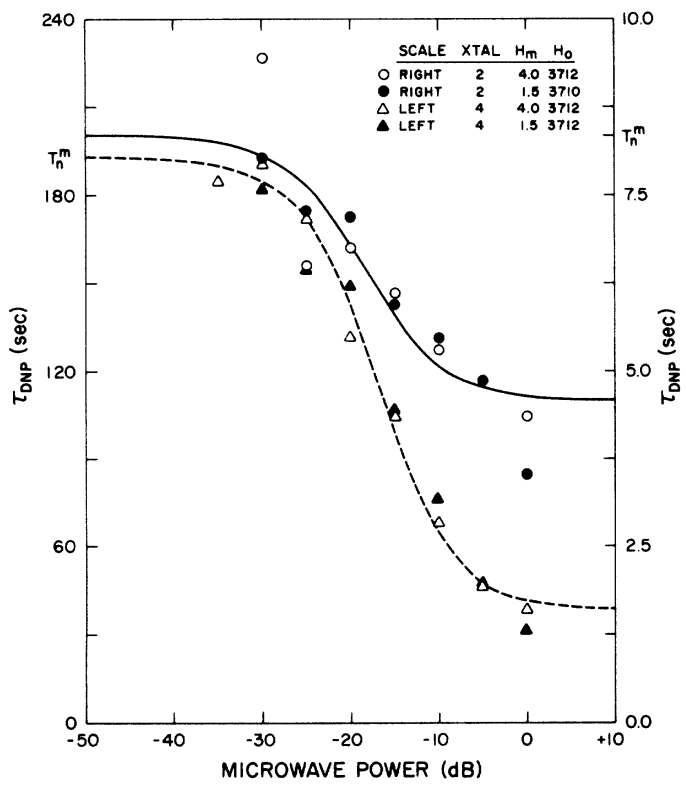

FIG. 10. DNP pump time $\tau_{\text {DNP }}$ Vs microwave power for crystals 2 and 4 at $H_{0} \approx 3712$ Oe and two $H_{m}$ values. Solid (broken) line is Eq. (4) for crystal 2 (3) using the best-fit parameters in Table III. Solid data points were not used in the least-squares fit.

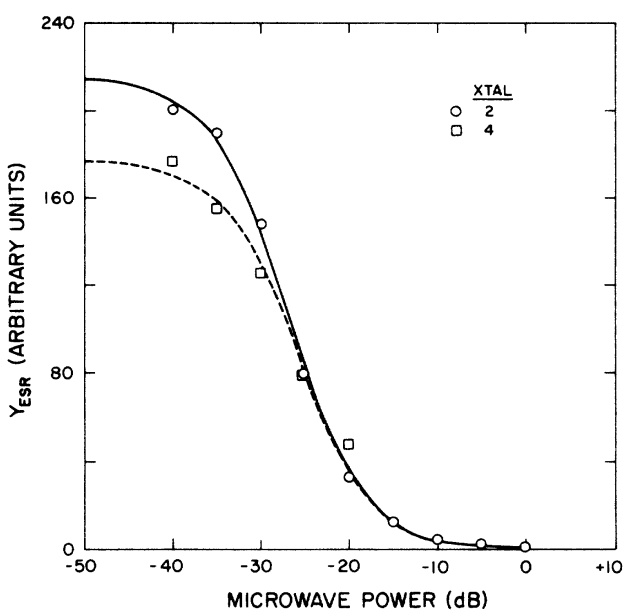

FIG. 11. ESR absorption derivative peak-to-peak signal height $Y_{\text {ESR }}$ vs microwave power for crystals 2 and 4. Solid (broken) line is Eq. (5) for crystal 2 (3) using the best-fit parameters in Table III.

absolute $E_{\mathrm{ss}}$ values and for $E_{\mathrm{ss}}$ vs $H_{0}$ line shapes, we obtain essentially the same results for crystals 1-3. The same conclusion is drawn from the $E_{\text {ss }}$ vs $P$ data in Figs. 4 and 7. It is only noteworthy that the $E_{\text {ss }}$ data points for crystals 3 and 4 in Fig. 7 do not flatten out at $P>-10 \mathrm{~dB}$ as do the data for crystal 2 in this figure and for crystal 1 in Fig. 4.

The $\tau_{\mathrm{DN} P}$ data points for crystal 3 at $P=0 \mathrm{~dB}$ in Fig. 8 lie above the Eq. (4) curve near $H_{0} \approx H_{00}$ $\approx 3700 \mathrm{Oe}$, and below Eq. (4) in the wings of the line. This effect is accentuated even more for crystal 2 in Fig. 9, where $\tau_{D N P}$ has a local maximum near $H_{0} \approx H_{00} \approx 3700 \mathrm{Oe}$, and minima near $H_{0} \approx H_{00} \pm \frac{1}{2} \Delta H_{p p}$, returning to $T_{n}^{m}$ in the far wings of the line. In an inverted $1 / \tau_{\mathrm{DNP}}$ vs $H_{0}$ plot, this would appear as a "dip" near $H_{00}$, and it will be referred to as the Fig. $91 / \tau_{D N P}$ dip, in this sense, henceforth. In Fig. 10 is shown $\tau_{D N P}$ vs $P$ data for crystals 2 and 4. Let $\tau_{D N P}^{\infty}$ be the infinite power DNP pump time at the $E_{\text {ss }}$ peaks, assuming a constant value for $\tau_{D N P}$ for $P \gg P_{1 / 2}$ at fixed $H_{0}$, as in Eq. (4). We estimate $\tau_{\mathrm{DN} P}^{\infty} \approx 4.0 \mathrm{sec}(36 \mathrm{sec})$ for crystals $2(4)$. That the ratio $T_{n}^{m} / \tau_{\mathrm{DNP}}^{\infty} \approx 2.1$ (5.4) differs for the two crystals $2(4)$, is related to the $1 / \tau_{\mathrm{DNP}}$ dip in Fig. 9 .

\section{DISCUSSION}

A. Theories in disagreement with DNP measurements

The strict shell-of-influence model of IHB SE DNP (Sec. II B of I) predicts (a) $E_{s s} \propto d G / d H$ at all $P$, (b) with $E_{s s}^{\text {peak }}$ reduced from $E_{i}$ by one or more orders of magnitude, and (c) with the NMR response of the polarized nuclei characterized in general by two time constants. In YES : ${ }^{168} \mathrm{Er}$ we found $E_{\mathrm{ss}} \propto d G / d H$ only at $P<P_{1 / 2}$. The measured 
ratio $E_{\mathrm{ss}}^{\text {peak }} / E_{i} \approx 0.2$ from Table II is to be compared with the strict shell-of-influence model prediction $E_{\mathrm{ss}}^{\text {peak }} / E_{i} \approx 0.02-0.08$ for crystals $1-3$ using Eq. (10) of I with $S \gg 1$ and the $\xi$ and $f_{e}^{m}$ values of Table III. Single time constant NMR response was observed under all circumstances (including, e.g., when changing $H_{0}$ in times $\left.\ll \tau_{D N P}\right)$. The strict shell-of-influence model is based on implausible theoretical assumptions, and receives no experimental confirmation in this work on YES : ${ }^{168} \mathrm{Er}$.

Cross-effect theory (Sec. III B of I) predicts (i) $E_{\mathrm{ss}} \propto\left[G\left(H_{0}\right)\right]^{2}(d G / d H)$, especially in the wings of the ESR line, (ii) with $E_{\mathrm{gs}}^{\text {peak }} / E_{i} \leq 0.1$, and (iii) with $\tau_{\mathrm{DNP}} \approx T_{n}^{m}$ for the unresolved CE. The $E_{\mathrm{ss}}$ vs $H_{0}$ data shown in Figs. 3, 5, and 6 fall off as $d G / d H$ or slower in the wings of the line, not faster, as in $C E$. The experimental ratio $E_{s s}^{\text {peak }} / E_{i} \approx 0.2$ is much larger than that obtained from Eq. (48) in I. In Figs. 8-10 we see $\tau_{\mathrm{DNP}} \neq T_{n}^{m}$ and, in fact, note $T_{n}^{m} / \tau_{\mathrm{DNP}}^{\infty} \approx 2.2,2.1$, and 5.4 , for crystals 1,2 , and 3 , respectively, which even violates $T_{n}^{m} / \tau_{\text {DNP }}$ $\leq 2$ [Eq. (34) of I] for the ideal case of the wellresolved CE. Thus CE processes are completely negligible compared to SE and EDDR DNP in these experiments.

The theoretical results of Buishvili et al. (Ref. 40 in I) predict a decrease in $E_{\mathrm{ss}}$ for IHB SE DNP with moderate spectral diffusion. The spectraldiffusion coefficient in that theory depends on $N_{e}$ in a complicated way, but the important point is that it is $N_{e}$ dependent. If their mechanism were to be effective in YES : ${ }^{168} \mathrm{Er}$, then $E_{\mathrm{ss}}$ would vary as the ${ }^{168} \mathrm{Er}$ dilution changes by a factor $\approx 20$ in crystals $1-4$. But $E_{\mathrm{ss}}$ is independent of $N_{e}$ (Table II). Hence the mechanism of Buishvili et al. finds no confirmation in this work on YES : ${ }^{168} \mathrm{Er}$.

\section{B. IHB SE DNP and the three-spectral-region model}

In order to examine the applicability of the threespectral-region model to these experiments, we have estimated some limits on ESR spectral diffusion in Appendix A. These estimates show that crystal 3 is IHB throughout most of its ESR spectrum, while crystal 2 is IHB in the wings and HB in the center of its ESR line. This suggests that the sizes of the SE, CE, and EDDR DNP regions in the three-spectral-region model (e.g., Fig. 8 of I) would be different for crystals $1-4$, which might lead to different $E_{\text {ss }}$ values. However, in Table II and in Figs. 3, 5, and 6, we see essentially identical $E_{s s}^{\text {peak }}$ values and $E_{s s}$ vs $H_{0}$ curves, so that no discrimination can be made with the $E_{\text {ss }}$ data.

The $\tau_{D N P}$ data are more enlightening. The crystal-3 $\tau_{D N P}$ data in Fig. 8 follow the Eq. (4) solid line moderately well. However, the $1 / \tau_{D N P}$ dip in Fig. 9 (crystal 2 ) is definitely suggestive of
HB behavior near the center of the ESR line, i. e., at $\left|H_{0}-H_{00}\right| \lesssim \frac{1}{2} \Delta H_{p p^{\circ}}$. A qualitative explanation for this dip might involve spectral diffusion of energy in the HB region I, leading to a decreased DNP rate, or actual EDDR DNP near $H_{00}$ at a rate $1 / \tau_{D N P}$ less than that of pure IHB $S E$ DNP. We can give no unique quantitative explanation. (Only one $\tau_{\mathrm{DNP}}$ data point was taken for crystal 1.) The progression from Fig. 8 to Fig. 9 as the ${ }^{168} \mathrm{Er}$ dilution goes from $0.01 \%$ to $0.05 \%$ is qualitatively consistent both with the three-spectralregion model and with Appendix A. The absence of any evidence for the CE mechanism in these experiments is consistent with a sharp transition from negligible to fast spectral diffusion in these IHB ESR lines, thus wiping out the CE region II. Such a sharp transition might also explain why the Buishvili et al. prediction is not verified in these experiments.

Let us summarize our results so far. The $E_{\mathbf{s s}}$, $\tau_{D N P}$, and $Y_{\text {ESR }}$ data points in Figs. 3-11 for crystals 1-3 fit the shapes of Eqs. (3)-(5) very well, except as noted. The best-fit parameters for $E_{\mathrm{ss}}$ and $Y_{\mathrm{ESR}}\left(\xi, S_{F 1}, S_{F 2}\right.$, and $\left.S_{F 6}\right)$ are all physically reasonable (except $\xi$ for crystals 1 and 2). The data are explicable in terms of the threespectral-region model assuming a negligible CE region II.

What are the difficulties with this analysis? First, the $E_{\mathrm{sg}}$ data at $P=0 \mathrm{~dB}$ in Figs. 3 and 6 fall off less rapidly than the theoretical Eq. (3) in the wings of the ESR line. This is crucial since $E_{\mathrm{ss}}$ vs $H_{0}$ curves for all DNP theories (with unresolved satellites) look essentially the same for $\left|H_{0}-H_{00}\right|$ $<\Delta H_{p p} / 2$, and therefore only the peak values $E_{\mathrm{ss}}^{\text {peak }}$ and the shape of the $E_{\mathrm{ss}}$ curve in the wings of the line are ordinarily us eful for discriminating different DNP theories. We saw in Sec. IIIA that YES : ${ }^{168} \mathrm{Er}$ probably satisfies $\tau \gg T_{n}^{m}$, so that crystals 1-3 are described by the muffin-tin model. But in the wings of an IHB ESR line, the muffintin model breaks down if a spin-diffusion criterion given in Ref. 4 is not satisfied. It appears that this may be the case in YES : ${ }^{168} \mathrm{Er}$, which complicates the analysis in an unknown way. ${ }^{4}$ While this may help explain the fact of an $E_{\mathrm{ss}}$ vs $H_{0}$ discrepancy in the wings of the lines, it does not confirm the theoretical predictions of Eq. (3).

Second, the best-fit values of the spin-packet width $\xi$ given in Table III are too large. The crystal-3 value of $\xi=3.19$ Oe is not outrageous in view of the crude rectangular spin-packet model underlying Eqs. (3)-(5), but $\xi=7.73$ Oe and $\xi$ $=18.9$ Oe in crystals 2 and 1 at first seem unreasonable. However, this is explicable in the three-spectral-region model, where the DNP is going from IHB SE in crystal 3 and perhaps the wings of crystal 2, to EDDR DNP at the $E_{\mathrm{ss}}$ peaks 
of crystal 2 and in crystal 1, as proposed above. Hence, $\xi$ should be reasonable in crystal 3 , but not necessarily so in crystals 1 and 2 .

Third, the scale factors $S_{F 3}-S_{F 5}$, which range from 1.47 to $9.18 \gg 1.0 \pm 0.2$, make no sense. Considerable effort was expended in using different combinations of scale factors, and more or fewer of them, to fit these data, but without success. We have no explanation for this.

Finally, we note that the magnetic field modulation is not expected to be an HB mechanis $m$ in these experiments since $f_{m}=97 \mathrm{~Hz} \ll T_{e}^{-1}\left(\approx 10^{4} \mathrm{sec}^{-1}\right)$, $\nu_{e} \approx 10^{10} \mathrm{~Hz}$. All our $E_{\mathrm{ss}}$ and $\tau_{\mathrm{DNP}}$ data are independent of $H_{m}$, in agreement with Eq. (8) of I.

\section{EDDR DNP theory}

To show that it is hard to discriminate IHB SE theory from EDDR DNP theory for an IHB ESR line with fast spectral diffusion, we now use the latter theory from Sec. IV of I to interpret the data presented in this paper. We assume $\left(T_{e} /\right.$ $\left.T_{d}\right) \omega_{L}^{2} \ll \omega_{\mathrm{IHB}}^{2}$. Then Eqs. (53) and (54) in I predict that for $S^{0}, S^{+}, S^{-} \gg 1$

$$
E_{\mathrm{ss}}^{\text {peak }}= \pm E_{i} \omega_{n} / 2 \omega_{\mathrm{IHB}}\left[1+\left(f_{e} \omega_{n}^{2} / \omega_{\mathrm{IHB}}^{2}\right)\right]^{1 / 2},
$$

at

$$
\Delta= \pm \omega_{\mathrm{IHB}}\left[1+\left(f_{e} \omega_{n}^{2} / \omega_{\mathrm{IHB}}^{2}\right)\right]^{1 / 2} .
$$

For a Gaussian $G(H)$, we have $\omega_{\text {IHB }}=\Delta H_{p p} / 2$, where $\omega_{\mathrm{IHB}} \approx 12$ Oe applies to these experiments. If $f_{e}=0$, Eqs. (6) and (7) then give $E_{\mathrm{ss}}^{\text {peak }} \approx \pm 144$ at $\Delta= \pm 12$ Oe. The largest possible $f_{e}$ from Table I is $f_{e} \approx 1$, in which case $\left[1+\left(f_{e} \omega_{n}^{2} / \omega_{\text {IHB }}^{2}\right)\right]^{1 / 2} \approx 1.12$, which changes Eqs. (6) and (7) to the estimates $E_{\text {ss }}^{\text {peak }} \approx \pm 129$ at $\Delta \approx \pm 13.4$ Oe, in agreement with Table II and Figs. 3 and $6 . E_{s s}$ is independent of $N_{e}$ in EDDR theory if $\left(T_{e} / T_{d}\right) \omega_{L}^{2} \ll \omega_{\mathrm{IHB}}^{2}$, but spectral diffusion remains fast. In this theory the shapes of Figs. 3 and 6 are not Lorentzian [see Eq. (53) in I] because of the falloff of $W^{0}$ in the wings of the ESR line.

That the shapes of $E_{\text {ss }}$ vs $H_{0}$ curves (for unresolved satellites) are similar in both IHB SE DNP and EDDR DNP theories has been shown before, ${ }^{14}$ and hence no attempt was made to leastsquares fit all the $E_{\text {ss }}$ data to EDDR DNP theory 。 Instead, it was hoped that the $\tau_{\mathrm{DN} P}$ data would allow the two theories to be discriminated. However, this does not work either. Consider the high-power $\left(S^{0}, S^{+}, S^{-} \gg 1\right)$ EDDR limit of $\tau_{\mathrm{DNP}}$, in particular, the short-dashed and solid lines in Fig. 7 of I, which represent Eqs. (59) and (61) of I. It can be shown that ${ }^{4} S^{d} f_{d} C_{n} \propto N_{e}$. Thus the $\tau_{\text {DNP }}$ data in Fig. 8 of this paper may be compared with the short-dashed line of Fig. 7 in I, while the Fig. $9 \tau_{\mathrm{DNP}}$ data here may represent an intermediate case between the short-dashed and solid curves in Fig. 7 of I. This progression has the right direction, since $N_{e}$ increases from crystal 3 to crystal 2 . In this connection, we note that $T_{n}^{m} / \tau_{\mathrm{DNP}}^{\infty} \approx 2.2(2.1)$ for crystals $1(2)$ is consistent with Eq. (62) in I, while the value $T_{n}^{m} / \tau_{\mathrm{DN} P}^{\infty} \approx 5.4$ for crystal 3 is not expected to obey Eq. (62) in I.

Thus the $E_{\mathrm{ss}}$ and $\tau_{\mathrm{DNP}}$ data measured in YES : ${ }^{168} \mathrm{Er}$ do not by themselves lead to a definite conclusion as to whether IHB SE DNP or EDDR DNP (with fast spectral diffusion) is the dominant mechanism. The only clearcut evidence for IHB SE in crystal 3 , and perhaps in the wings of crystal 2 , is the order-of-magnitude estimate $T_{e} /\left(T_{2 e}^{B}\right)_{i}<1$ in Appendix A, which makes IHB SE DNP highly probable there, and lends credence to some version of the three-spectral-region model (with negligible $\mathrm{CE}$ ). Of course, spectral diffusion may not be significant when $\left(T_{2 e}^{B}\right)_{i}$ is larger than $T_{e}$ by only a small amount, so IHB SE may be dominant in the wings of crystal 1 , too. This latter view is supported by the $1 / T_{n}^{m}$ data in Fig. 2, which suggest that all three crystals 1-3 have the same size $\mathrm{HB}$ region $I$ in the three-spectral-region model. Evidence against this interpretation is the $1 / \tau_{\mathrm{DN} P}$ dip in Fig. 9 and the large values of $\xi$ for crystals 1 and 2 in Table III.

To decide conclusively the issues raised in this paper would require further experimental work on YES : ${ }^{168} \mathrm{Er}$ at ${ }^{168} \mathrm{Er}$ concentrations below $0.01 \%$ and above $0.2 \%$, which would put this system into the pure IHB and pure EDDR regimes, respectively.

The limited $T_{n}^{m}$ data in Fig. 2 can be explained qualitatively either by the three-spectral-region model or by EDDR relaxation. ${ }^{4}$ Figure 1 of this paper indicates how application of the threespectral-region model to $T_{n}^{m}$ might be tested. As $\theta$ is varied from $\approx 8^{\circ}$ to $0^{\circ}, \Delta H_{p p}$ collapses from $\approx 27$ Oe to $\approx 5 \mathrm{Oe}$, thus enhancing the HB region $I$ in this model. Measurements of $T_{n}^{m}$ as a function of $H_{0}, T, N_{e}$, and $\theta\left(0^{\circ}<\theta \leqslant 8^{\circ}\right)$ could be fit to phenomenological theories for region $I$. YES : ${ }^{168} \mathrm{Er}$ near $\theta \approx 0^{\circ}$ is a useful system for such studies because of its large $g_{1}$ and because of the large change in $\Delta H_{p p}$ as $\theta$ is varied by small amounts near $\theta \approx 0^{\circ}$ where the anisotropic EDDR theory of $I$ is approximately valid.

\section{SUMMARY}

Results of DNP and ESR experiments in six YES : ${ }^{168} \mathrm{Er}$ crystals are reported here. DNP peak enhancements $E_{\mathbf{s s}}^{\text {peak }} \approx 120$ are obtained for crystals $1-3$ having ${ }^{168} \mathrm{Er}$ dilutions of $0.2 \%-0.01 \%$. The $E_{\text {ss }}$ vs $H_{0}$ curves are proportional to the ESR absorption derivative $d G / d H$ at low powers $(\approx 0.3$ $\mathrm{mW}$ ), but change shape in the wings of the ESR line at high powers $(\approx 300 \mathrm{~mW})$. Essentially identical $E_{\text {ss }}$ data are obtained for all three crystals. Extensive DNP pump time $\tau_{\mathrm{DN} P}$ and ESR data for crystals 2-4, as well as limited data for 
the other crystals, are also given. No $T_{e}$ measurements were made here, as we utilized Larson and Jeffries's ${ }^{6}$ results for YES : Er.

There is no $E_{\mathrm{ss}}$ or $\tau_{\mathrm{DNP}}$ evidence in these experiments (a) for the traditional strict shell-ofinfluence model of IHB SE DNP, (b) for nonnegligible CE DNP processes, or (c) for decreases in $E_{\text {ss }}$ due to moderate spectral diffusion in an IHB ESR line, as proposed by Buishvili et al. (Ref. 40 of I).

The DNP and ESR data of crystals 1-3 have been fit by the least-squares technique to the muffintin-model equations of IHB SE DNP given in I. Moderate agreement with theory is obtained, especially if the data are interpreted with the threespectral-region model given in I for wide IHB ESR lines. These data have also been analyzed in terms of EDDR DNP theory for an IHB ESR line with fast spectral diffusion throughout the line. There is qualitative and some quantitative agreement between this theory and the data. It is thus difficult to discriminate EDDR DNP theory from IHB SE DNP with the three-spectral-region model in these experiments. The only clearcut evidence for the latter is an order-of-magnitude theoretical calculation of the electron magnetic dipole-dipole contribution to the ESR intrinsic spin-packet width, which gives non-negligible spectral diffusion in the centers of the crystal-1 and - 2 ESR lines, and negligible spectral diffusion in the wings of crystals 3 and (perhaps) 2. Conclusive evidence would require further experiments at ${ }^{168} \mathrm{Er}$ dilutions lower than $0.01 \%$ and higher than $0.2 \%$. The YES : ${ }^{168} \mathrm{Er}$ system at $\theta \approx 0^{\circ}-8^{\circ}$ is useful for further tests of the three-spectral-region model because of the large change in $\Delta H_{p p}$ for small $\theta$ variations.

Finally, we note $E_{\mathbf{s s}}^{\text {peak }} \approx 460-500$ for crystals $1-3$ from Eq. (3) if $f_{e}^{m}=0$, but all the remaining parameters from Table III are used. This gives $E_{\mathrm{ss}}^{\text {peak }} / E_{i} \approx 0.80-0.87$. Hence IHB SE DNP may be us eful for polarized nuclear targets ${ }^{3,8,9}$ in favorable cases, contrary to the traditional view of the strict shell-of-influence model. Since $f_{e}^{m}$ often decreases at higher $H_{0}$, it would be us eful to test YES : ${ }^{168} \mathrm{Er}$ at $\nu_{e} \approx 35,50$, or $70 \mathrm{GHz}$ for large $E_{s s}^{\text {peak }} \approx E_{i}$. This has not been done here since only 9.5-GHz equipment is available in this laboratory. YES : ${ }^{168} \mathrm{Er}$ is a poor polarized nuclear target material because of its low hydrogen content, but its ESR properties make it us eful for tests of high DNP by IHB SE.

\section{ACKNOWLEDGMENTS}

I would like to thank R. A. Arndt for his help with the computer programs. J. W. Poulton and $\mathrm{H}$. C. Ballagh, Jr. gave enthusiastic aid in the early stages of this work. Helpful conversa- tions and/or correspondence with C. D. Jeffries, J. P. Wolfe, H. J. Stapleton, G. Feher, and E. A. Gere are gratefully acknowledged.

\section{APPENDIX A: LIMITS ON ESR SPECTRAL DIFFUSION}

We estimate theoretically the spin-packet width $\xi$, and then use it to set some limits on ESR spectral diffusion. As discussed in Sec. IV B, the magnetic field modulation is not an HB mechanism in these experiments. Hence $\xi$ is given by $\mathrm{Eq}$.

(8) of I, which we rewrite as

$$
\xi \approx\left[H_{\mathrm{in}}^{2}+\left(2 H_{1}\right)^{2}\right]^{1 / 2} .
$$

The microwave field amplitude $H_{1}$ is easily estimated from the rule of thumb ${ }^{15}$ that $1 \mathrm{~W}$ of $X$-band power gives $H_{1}=1$ Oe with a cavity $Q \approx 10^{3}$. The maximum microwave cavity power $\approx 0.3 \mathrm{~W}$ used here gives a peak-to-peak amplitude $2 H_{1} \approx 0.6 \mathrm{Oe}$, leading to the relation $0.6 \geq 2 H_{1} \geq 0$ Oe in these experiments. The two principal contributors to the intrinsic packet width $H_{\text {in }}$ are $T_{e}$ and $T_{2 e}$ processes. Using Larson and Jeffries's ${ }^{6}$ value of $T_{e}=8 \times 10^{-5} \mathrm{sec}$ measured in their YES : Er crystals, we obtain a $T_{e}$ contribution of $H_{\text {in }} \approx 2 \times 10^{-3}$ Oe for the conditions of these experiments.

The $T_{2 e}$ and spectral-diffusion calculation is more complicated. Estimates of $2 \Delta_{B}$ and $T_{2 e}^{B}$ based on Appendix A of I are given for crystals $1-3$ in Table $\mathrm{V}$ for $\theta=0^{\circ}$. (The values for $\theta=6.1^{\circ}$ would differ only slightly from those in Table $V_{\circ}$ ) Since $2\left(g_{11} / g_{1}\right)^{2} \approx 0.06$ for YES : Er, the width $\Delta_{1 / 2}$ at $\theta \approx 0^{\circ}$ is dominated by the $\Delta_{B}$ term. The equations for $2 \Delta_{B}$ and $T_{2 e}^{B}$ in the Kittel and Abrahams theory are valid for a completely $\mathrm{HB}$ line with no IHB mechanisms, and hence represent only limits for a given spin packet in an IHB line. Since $2 \Delta_{B}$ in Table $\mathrm{V}$ is up to 100 times smaller than $\Delta H_{\mathrm{pp}}$ in Table I, YES : ${ }^{168} \mathrm{Er}$ is close to IHB at the lower ${ }^{168} \mathrm{Er}$ dilutions.

For a completely IHB line we can calculate

$$
\left(T_{2 e}^{B}\right)_{i}=T_{2 e}^{B} / \xi G\left(H_{i}\right)
$$

TABLE V. Comparison of the empirically estimated spin-packet width $\xi$ with the theoretical linewidth $2 \Delta_{B}$ from the Kittel and Abrahams theory for a purely HB ESR line. Estimates of $T_{2 e}^{B}$ from their theory are also included.

\begin{tabular}{lccc}
\hline \hline & Crystal 1 & Crystal 2 & Crystal 3 \\
\hline$\xi\left(\right.$ Oe $^{\mathrm{a}}$ & 18.9 & 7.7 & 3.2 \\
$2 \Delta_{B}(\mathrm{Oe})^{\mathrm{b}}$ & 5.7 & 1.4 & 0.29 \\
$T_{2 e}^{\mathrm{B}}(\mathrm{sec})^{\mathrm{b}}$ & $2.7 \times 10^{-8}$ & $1.1 \times 10^{-7}$ & $5.3 \times 10^{-7}$ \\
\hline \hline
\end{tabular}

${ }^{\mathrm{a}}$ From Table III. $\quad$ 'See text. 
for the spin packet at $H_{i}$ if $T_{e} \ll\left(T_{2 e}^{B}\right)_{i}$, as in I. This calculation can be done self-consistently, as follows. Estimate an upper limit $\xi_{\max }$ from Eq. (7) or (8) in I, using $H_{1}, T_{e}, T_{2 e}^{B}$, and $H_{m}$ (if relevant), to check that $\xi_{\max }$ is much smaller than the measured ESR $\Delta H_{p p}$. Then calculate $\left(T_{2 e}^{B}\right)_{i}$ from Eq. (A2) using $\xi=\xi_{\max }$. If the condition $T_{e}$ $\ll\left(T_{2 e}^{B}\right)_{i}$ holds in this worst case analysis, then Eq. (A2) is valid and one can safely predict that (i) $\xi$ is independent of $\left(T_{2 e}^{B}\right)_{i}$ at $H_{i}$, and (ii) spectral diffusion in the ESR line near $H_{i}$ is negligible. The latter is true because the electrons at $H_{i}$ are more tightly coupled to the lattice than to each other. Since even order-of-magnitude estimates of spectral-diffusion times are difficult, ${ }^{15}$ the condition $\left(T_{2 e}^{B}\right)_{i}>T_{e}$ does not by itself indicate fast spectral diffusion in an IHB line, although one must be wary in this case.

Let us apply this scheme to the wings of crystal
3, where we define the wings of an ESR line to correspond to $\left|H_{0}-H_{00}\right| \geq \Delta H_{\mathrm{pp}}$ at fixed $\nu_{e}$. Since $2 \Delta_{B} \approx 0.3$ Oe in crystal 3 (Table V), we take $\xi_{\max }$ $\approx 2 H_{1} \approx 0.6$ Oe $\ll \Delta H_{p p}$. Using a Gaussian $G(H)$ with $\Delta H_{\mathrm{pp}}=24 \mathrm{Oe}$, we calculate $\left(T_{2 e}^{B}\right)_{i}=2 \times 10^{-4} \mathrm{sec}$ $>T_{e}=0.8 \times 10^{-4} \mathrm{sec}$ at $\left|H_{0}-H_{00}\right|=\Delta H_{\mathrm{pp}}$. Hence we find $H_{\text {in }} \approx 2 \times 10^{-3}$ Oe $\propto 1 / T_{e}$ in the wings of crystal 3 . If the $0.01 \%{ }^{168} \mathrm{Er}$ dilution used here is in fact high by a factor $\approx 5$, or if $T_{e}$ is smaller than that measured in Ref. 6, then this result would apply to almost the entire ESR line of crystal 3 and to the wings of crystal 2, which would all be IHB with negligible spectral diffusion. Using $\xi_{\max }$ $\approx 0.6 \mathrm{Oe}$, we calculate $\left(T_{2 e}^{B}\right)_{i}<T_{e}$ for crystal 1 and for the center of the crystal-2 ESR line, so no definite theoretical prediction about fast (or slow) spectral diffusion can be made. Nevertheless, it seems plausible that $H_{\text {in }} \propto\left(T_{2 e}^{B}\right)_{i}^{-1}$ may satisfy $H_{\text {in }} \ll 2 \Delta_{B} \ll \Delta H_{p p}$ in crystals 1 and 2 .

Temperature Physics, edited by C. J. Gorter (NorthHolland, Amsterdam, 1964), Vol. 4, pp. 384-449.

${ }^{9} \mathrm{C}$. D. Jeffries, in Electron Paramagnetic Resonance, edited by S. Geschwind (Plenum, New York, 1972), pp. 217-262.

${ }^{10}$ P. L. Scott, H. J. Stapleton, and C. Wainstein, Phys. Rev. 137, A 71 (1965)

${ }^{11}$ Developed by E. A. Gere (private communication). Professor G. Feher kindly made a sample available to this laboratory.

${ }^{12} \mathrm{~J}$. P. Wolfe and C. D. Jeffries, Phys. Rev. B 4, 731 (1971).

${ }^{13}$ C. E. Byvik and D. S. Wollan, Phys. Rev. B 10,791 (1974).

${ }^{14}$ H. H. Niebuhr, E. E. Hundt, and E. Brun, Helv. Phys. Acta 43, 777 (1970).

${ }^{15}$ W. B. Mims, Ref. 9, pp. 263-351. See p. 325 for the relation between $H_{1}$ and the microwave power $P$. 